PROCEEDINGS OF THE

AMERICAN MATHEMATICAL SOCIETY

Volume 140, Number 7, July 2012, Pages 2537-2541

S 0002-9939(2011)11119-5

Article electronically published on October 28, 2011

\title{
A COINCIDENCE THEOREM FOR COMMUTING INVOLUTIONS
}

\author{
PEDRO L. Q. PERGHER
}

(Communicated by Alexander N. Dranishnikov)

\begin{abstract}
Let $M^{m}$ be an $m$-dimensional, closed and smooth manifold, and let $S, T: M^{m} \rightarrow M^{m}$ be two smooth and commuting diffeomorphisms of period 2. Suppose that $S \neq T$ on each component of $M^{m}$. Denote by $F_{S}$ and $F_{T}$ the respective sets of fixed points. In this paper we prove the following coincidence theorem: if $F_{T}$ is empty and the number of points of $F_{S}$ is of the form $2 p$, with $p$ odd, then $\operatorname{Coinc}(S, T)=\left\{x \in M^{m} \mid S(x)=T(x)\right\}$ has at least some component of dimension $m-1$. This generalizes the classic example given by $M^{m}=S^{m}$, the $m$-dimensional sphere, $S\left(x_{0}, x_{1}, \ldots, x_{m}\right)=$ $\left(-x_{0},-x_{1}, \ldots,-x_{m-1}, x_{m}\right)$ and $T$ the antipodal map.
\end{abstract}

\section{INTRODUCTION}

Coincidence theory concerns itself with the following basic question: given two maps $f, g: X \rightarrow Y$ of topological spaces, when is there a coincidence point, that is, a point $x \in X$ such that $f(x)=g(x)$ ? If so, another question is concerned with the size of the set of coincidence points, $\operatorname{Coinc}(f, g)$. For example, one direction is looking for maps $f^{\prime}, g^{\prime}: X \rightarrow Y$, with $f^{\prime}$ homotopic to $f$ and $g^{\prime}$ homotopic to $g$, so that, in some sense, the size of $\operatorname{Coinc}\left(f^{\prime}, g^{\prime}\right)$ is minimal. Coincidence theory includes the Fixed Point theory, with $X=Y$ and $g$ the identity map. Also, it includes questions of Borsuk-Ulam type: if $X$ is equipped with a free involution $T: X \rightarrow X$, one asks for $\operatorname{Coinc}(f, f T)$. As is well known, there is a lot of articles in the literature dealing with such questions, and it is unnecessary to list references.

In the generality under which the coincidence question above is stated, one can say almost nothing of interest; good questions arise when we focus attention on specific situations. In this setting, consider $X=Y=M^{m}$ to be an $m$-dimensional, closed and smooth manifold, and $S$ and $T$ two smooth commuting diffeomorphisms of finite period on $M^{m}$. If $T: M^{m} \rightarrow M^{m}$ is a smooth diffeomorphism of finite period, the fixed point set of $T, F_{T}$, is either empty or a finite and disjoint union of closed submanifolds of $M^{m}, F_{T}=\bigcup_{j=0}^{n} F^{j}(n<m)$, where $F^{j}$ denotes the union of those components of $F_{T}$ having dimension $j$ (see [1, page 206]). We denote by $\operatorname{dim}\left(F_{T}\right)$ the maximum dimension of a component

Received by the editors December 6, 2010 and, in revised form, February 16, 2011.

2010 Mathematics Subject Classification. Primary 55M20; Secondary 57R75, 57R85.

Key words and phrases. Coincidence point, involution, characteristic class, projective space bundle, characteristic number, singular manifold, unoriented cobordism class, equivariant diffeomorphism.

The author was partially supported by CNPq and FAPESP. 
of $F_{T} ; \operatorname{dim}\left(F_{T}\right)$ is a reasonable parameter to measure the size of $F_{T}$. Here, we are excluding components of $M^{m}$ on which $T$ acts as the identity map. Note that $\operatorname{Coinc}(S, T)$ is the fixed point set of $S^{-1} T$, which is a diffeomorphism with period the least common multiple of the periods of $S$ and $T$. We raise the following specific coincidence question: to what extent can $\operatorname{dim}(S)$ and $\operatorname{dim}(T)$ influence $\operatorname{dim}\left(S^{-1} T\right)$ ? In this paper we deal with a particular case of this question, taking $S$ and $T$ with period 2, that is, with $S$ and $T$ being involutions. Our result is motivated by the following classic example: consider $S, T: S^{m} \rightarrow S^{m}$, where $S^{m}$ is the $m$-dimensional sphere, $S\left(x_{0}, x_{1}, \ldots, x_{m}\right)=\left(-x_{0},-x_{1}, \ldots,-x_{m-1}, x_{m}\right)$ and $T\left(x_{0}, x_{1}, \ldots, x_{m}\right)=\left(-x_{0},-x_{1}, \ldots,-x_{m}\right)$ (the antipodal map). Then $F_{S}$ consists of two points, $F_{T}$ is empty and $\operatorname{dim}\left(F_{S T}\right)=m-1$. We will see that in a much more general situation, the behavior is very similar to the classic example. Specifically, we will prove the following:

Theorem. Let $M^{m}$ be an m-dimensional, closed and smooth manifold, and $S$ and $T$ two smooth and commuting involutions on $M^{m}$ with $S \neq T$ on each component of $M^{m}$. Suppose that $F_{T}$ is empty and the number of points of $F_{S}$ is of the form $2 p$, with $p$ odd. Then $\operatorname{Coinc}(S, T)$ has at least some component of dimension $m-1$; that is, $\operatorname{dim}\left(F_{S T}\right)=m-1$.

Remark 1. As an example of $S: M^{m} \rightarrow M^{m}$ with $2 p$ fixed points, take the disjoint union of $p$ copies of the above described $S: S^{m} \rightarrow S^{m}$.

Remark 2. The result is not valid if $F_{S}$ has $2 p$ points with $p$ not odd. In fact, consider again the involutions of the classic example, $S, T: S^{m} \rightarrow S^{m}$. Let $\left(S^{m}\right)^{r}$ be the Cartesian product of $r$ copies of $S^{m}$, where $r \geq 2$. On $\left(S^{m}\right)^{r}$ we consider the product involutions $\mathcal{S}=S \times S \times \ldots \times S$ and $\mathcal{T}=T \times T \times \ldots \times T(r$ times $)$. Then $F_{\mathcal{T}}$ is empty and $F_{\mathcal{S}}$ has $2^{r}$ points; however, $\operatorname{dim}\left(F_{\mathcal{S} \mathcal{T}}\right)=m r-r \neq m r-1$.

Remark 3. The above theorem suggests that some kind of compensation law determining the possibilities for $\operatorname{dim}\left(F_{S}\right), \operatorname{dim}\left(F_{T}\right)$ and $\operatorname{dim}\left(F_{S T}\right)$ could be valid (for example, $\left.\operatorname{dim}\left(F_{S}\right)+\operatorname{dim}\left(F_{T}\right)+\operatorname{dim}\left(F_{S T}\right) \geq m-1\right)$. However, in general there need be no relation among the three dimensions. In fact, one may consider any involution $U: N^{n} \rightarrow N^{n}$ on a closed smooth $n$-dimensional manifold $N^{n}$ and let $M=N^{n} \times S^{j}$ with $S=U \times I d, T=I d \times-I d$; here $j \geq 1$ and $I d$ is the identity map. Then $F_{T}$ and $F_{S T}$ are empty and $F_{S}=F_{U} \times S^{j}$. Special choices of $j$ and $\left(N^{n}, U\right)$ give an essentially arbitrary choice of dimensions.

\section{Proof of the Result}

The main tool to be used lies in the context started with the famous work [3] of R. Thom about cobordism theory, which gave him the Fields Medal in 1958, and continued ten years later with the monumental work [2] of P. Conner and E. Floyd, which extended the previous Thom results to the setting of singular cobordism of spaces.

Write $F_{S}=\left\{P_{1}, P_{2}, \ldots, P_{2 p}\right\}, F_{S T}=\bigcup_{j=0}^{n} F^{j} ;$ at this stage, $F_{S T}$ could be empty. Because $T$ is free, $P_{i} \notin F^{j}$, for every $i=1,2, \ldots, 2 p$ and $j=0,1, \ldots, n$. Because of the commutativity of the involutions, $S T$ acts freely on $F_{S}$ and $S$ acts freely on $F_{S T}$. Without loss, we can suppose that $S T\left(P_{2 i-1}\right)=P_{2 i}, i=1,2, \ldots, p$. Choose disjoint closed $m$-balls $B_{1}, B_{2}, \ldots, B_{2 p}$ with center $P_{1}, P_{2}, \ldots, P_{2 p}$, respectively. The 
radius $\epsilon>0$ of these balls can be selected so that: i) each $B_{i}$ is invariant under $S$, and up to an equivariant diffeomorphism $S$ acts on $B_{i}$ as the antipodal map; ii) $S T$ acts on $B_{1} \cup B_{2} \cup \ldots \cup B_{2 p}$ as an orthogonal bundle map covering the action of $S T$ on $F_{S}$. That is, each $B_{2 i-1}$ is orthogonally mapped by ST into $B_{2 i}$. Denote by $\eta_{j} \rightarrow F^{j}$ the normal bundle of $F^{j}$ in $M^{m}, j=0,1, \ldots, n$, and by $D\left(\eta_{j}\right)$ the associated disk bundle. By identifying disk bundles associated to normal bundles to suitable tubular neighborhoods of the base spaces, each $D\left(\eta_{j}\right)$ is identified to a tubular neighborhood $D_{j}$ of $F^{j}$ so that $\bigcup_{j=0}^{n} D_{j}$ is the union of a pairwise disjoint collection of tubular neighborhoods of the various components of $F_{S T}$. Again, the radius $\epsilon>0$ of these tubular neighborhoods can be selected so that: i) $D_{j} \cap B_{i}=\emptyset$ for every $j=0,1, \ldots, n$ and $i=1,2, \ldots, 2 p$; ii) each $D_{j}$ is invariant under $S T$, and up to an equivariant diffeomorphism $S T$ acts on each fiber of $D_{j}$ as the antipodal map; iii) each $D_{j}$ is invariant under $S$, and up to an equivariant diffeomorphism $S$ acts on $D_{j}$ as an orthogonal bundle map covering the action of $S$ on $F^{j}$. These choices are made possible by the fact that $S$ and $S T$ define a smooth $Z_{2} \oplus Z_{2}$-action on $M^{m}$, and thus there is a Riemannian metric on $M^{m}$ with respect to which $S$ and $S T$ act as isometries; see [2, Chapter 3, Section 22, pages 57 and 58].

Consider the manifold $W$ obtained by removing the interior of all these tubular neighborhoods from $M^{m}$. That is,

$$
W=M^{m}-\left(\left(\bigcup_{i=1}^{2 p} \operatorname{int}\left(B_{i}\right)\right) \cup\left(\bigcup_{j=0}^{n} \operatorname{int}\left(D_{j}\right)\right)\right) ;
$$

$W$ is an $m$-dimensional and compact manifold with boundary

$$
\partial(W)=\left(\bigcup_{i=1}^{2 p} \partial\left(B_{i}\right)\right) \cup\left(\bigcup_{j=0}^{n} \partial\left(D_{j}\right)\right) .
$$

Call $W^{\prime}=\frac{W}{S T}$ the orbit space of $W$ by $S T$. Since $S T$ acts freely on $W, W^{\prime}$ is an $m$-dimensional and compact manifold with boundary

$$
\partial\left(W^{\prime}\right)=\frac{\partial(W)}{S T}
$$

Call $W^{\prime \prime}=\frac{W^{\prime}}{S}$. Since $S$ and $T$ act freely on $W, S$ acts freely on $W^{\prime}$, so $W^{\prime \prime}$ is an $m$-dimensional and compact manifold with boundary

$$
\partial\left(W^{\prime \prime}\right)=\frac{\partial\left(W^{\prime}\right)}{S}
$$

Set $\mu \rightarrow W^{\prime \prime}$ for the line bundle of the double cover $W^{\prime} \rightarrow W^{\prime \prime}$, and take $F: W^{\prime \prime} \rightarrow$ $B O(1)$ a classifying map of this bundle. Set

$$
f=F_{\mid \partial\left(W^{\prime \prime}\right)}: \partial\left(W^{\prime \prime}\right) \rightarrow B O(1),
$$

the restriction map. Write $\xi \rightarrow \partial\left(W^{\prime \prime}\right)$ for the restriction of the bundle $\mu$ to $\partial\left(W^{\prime \prime}\right)$; obviously, $f$ is a classifying map of $\xi$. Following Conner and Floyd, the pair $\left(\partial\left(W^{\prime \prime}\right), f\right)$ is a singular manifold in $B O(1)$ and represents an unoriented cobordism class $\left[\left(\partial\left(W^{\prime \prime}\right), f\right)\right]$ in the $(m-1)$-dimensional unoriented cobordism group of $B O(1)$, $\mathcal{N}_{m-1}(B O(1))$; see [2, Chapter 1, Section 4]. Denoting the generator of the first cohomology group $H^{1}\left(B O(1), Z_{2}\right)$ by $c$, the characteristic class of $\xi \rightarrow \partial\left(W^{\prime \prime}\right)$ is then

$$
w_{1}(\xi)=f^{*}(c) \in H^{1}\left(\partial\left(W^{\prime \prime}\right), Z_{2}\right) .
$$


Because $\partial\left(W^{\prime \prime}\right)$ is a boundary and the restriction of $F$ is $f,\left[\left(\partial\left(W^{\prime \prime}\right), f\right)\right]$ bounds as a singular manifold in $B O(1)$; see [2, Chapter 1, Section 4]. Therefore the Whitney number obtained by evaluating the cohomology class

$$
f^{*}\left(c^{m-1}\right)=\left(w_{1}(\xi)\right)^{m-1} \in H^{m-1}\left(\partial\left(W^{\prime \prime}\right), Z_{2}\right)
$$

on the fundamental homology class $\left[\partial\left(W^{\prime \prime}\right)\right]$,

$$
\left(w_{1}(\xi)\right)^{m-1}\left[\partial\left(W^{\prime \prime}\right)\right]
$$

gives a (modulo 2) zero characteristic number; see [2, Theorem 17.2, page 47]. The next step is to analyze more closely this characteristic number, and the first analysis is over $F_{S}$. Note that $\frac{\bigcup_{i=1}^{2 p} \partial\left(B_{i}\right)}{S T}$ is the disjoint union of $p$ spheres of dimension $m-1$,

where

$$
\frac{\bigcup_{i=1}^{2 p} \partial\left(B_{i}\right)}{S T}=S_{1}^{m-1} \cup S_{2}^{m-1} \cup \ldots \cup S_{p}^{m-1}
$$

$$
S_{i}^{m-1}=\frac{\partial\left(B_{2 i-1}\right) \cup \partial\left(B_{2 i}\right)}{S T} .
$$

Since $S$ acts on each $\partial\left(B_{i}\right)$ as the antipodal map, it acts on each $S_{i}^{m-1}$ in the same way; in this way, the part of $\partial\left(W^{\prime \prime}\right)$ corresponding to $F_{S}$ is the union of $p$ real projective spaces of dimension $m-1$,

$$
\frac{S_{1}^{m-1}}{S} \cup \frac{S_{2}^{m-1}}{S} \cup \ldots \cup \frac{S_{p}^{m-1}}{S} .
$$

If $\alpha_{i} \in H^{1}\left(\frac{S_{i}^{m-1}}{S}, Z_{2}\right) \cong Z_{2}$ is the generator, it is known that $\alpha_{i}$ is the characteristic class of the double cover $S_{i}^{m-1} \rightarrow \frac{S_{i}^{m-1}}{S}$ and $\alpha_{i}^{m-1} \in H^{m-1}\left(\frac{S_{i}^{m-1}}{S}, Z_{2}\right) \cong Z_{2}$ is the generator. Since $p$ is odd, it follows that, on $F_{S}$, the characteristic number $\left(w_{1}(\xi)\right)^{m-1}\left[\partial\left(W^{\prime \prime}\right)\right]$ becomes

$$
\sum_{i=1}^{p} \alpha_{i}^{m-1}\left[\frac{S_{i}^{m-1}}{S}\right]=1 .
$$

Hence some component of $F_{S T}$ must provide a nonzero characteristic number, which in particular implies that $F_{S T}$ is nonempty. Take $j<m-1$; we will analyze $\left(w_{1}(\xi)\right)^{m-1}\left[\partial\left(W^{\prime \prime}\right)\right]$ on $F^{j}$. Note that $\frac{\partial\left(D_{j}\right)}{S T}$ is the total space of the real projective space bundle $p_{j}: R P\left(\eta_{j}\right) \rightarrow F_{j}$ associated to $\eta_{j}, p_{j}$ the projection map. Further, $S$ acts as a bundle map and freely on $R P\left(\eta_{j}\right)$, and this action covers the free action of $S$ on $F_{j}$. Hence $p_{j}$ induces the projection map

$$
p_{j}^{\prime}: \frac{R P\left(\eta_{j}\right)}{S} \rightarrow \frac{F_{j}}{S} .
$$

Write $\xi_{j} \rightarrow \frac{R P\left(\eta_{j}\right)}{S}$ for the restriction of the line bundle $\xi \rightarrow \partial\left(W^{\prime \prime}\right)$ to $\frac{R P\left(\eta_{j}\right)}{S}$. Denoting by $\nu \rightarrow \frac{F_{j}}{S}$ the line bundle of the double cover $F_{j} \rightarrow \frac{F_{j}}{S}$, one has that $\xi_{j} \rightarrow \frac{R P\left(\eta_{j}\right)}{S}$ is the pullback

$$
p_{j}^{\prime *}(\nu) \rightarrow \frac{R P\left(\eta_{j}\right)}{S} .
$$


So

$$
\left(w_{1}\left(\xi_{j}\right)\right)^{m-1}=\left(p_{j}^{\prime *}\left(w_{1}(\nu)\right)\right)^{m-1}=p_{j}^{\prime *}\left(w_{1}(\nu)^{m-1}\right) \in H^{m-1}\left(\frac{R P\left(\eta_{j}\right)}{S}, Z_{2}\right) .
$$

Because $w_{1}(\nu)^{m-1} \in H^{m-1}\left(F_{j}, Z_{2}\right)$ and $m-1>j$, one has that $w_{1}(\nu)^{m-1}=0$. It follows that, on $\bigcup_{j=0}^{m-2} F^{j}$, the characteristic number $\left(w_{1}(\xi)\right)^{m-1}\left[\partial\left(W^{\prime \prime}\right)\right]$ is zero. Therefore some component of $F^{m-1}$ must provide a nonzero characteristic number, which ends the proof.

Remark. Taking into account our result and Remarks 2 and 3, we raise the question of finding conditions on the pairs $\left(M^{m}, T\right),\left(M^{m}, S\right)$, under which some relation among the three dimensions could be valid (e.g., the one stated in Remark 3). We also ask for similar results when $S$ and $T$ are diffeomorphims with other periods.

\section{ACKNOWLEDGEMENT}

I would like to thank the referee for suggestions that helped to clarify the original version.

\section{REFERENCES}

1. D. Montgomery and L. Zippin, Topological Transformation Groups, Interscience Publishing Co., 1955. MR0073104 (17:383b)

2. P. E. Conner and E. E. Floyd, Differentiable Periodic Maps, Springer-Verlag, Berlin, 1964. MR0176478(31:750)

3. R. Thom, Quelques propriétés globales des variétés differentiables, Comment. Math. Helv. 28 (1954), 18-88. MR0061823(15:890a)

Departamento de Matemática, Universidade Federal de São Carlos, Caixa Postal 676, CEP 13.565-905, SÃo CARLOS, SP, BraZIL

E-mail address: pergher@dm.ufscar.br 